\title{
Dimension-Dependent Upper Bounds for Grebner Bases
}

\author{
Amir Hashemi \\ Department of Mathematical Sciences \\ Isfahan University of Technology \\ Isfahan, 84156-83111, Iran \\ School of Mathematics, Institute for Research in \\ Fundamental Sciences (IPM) \\ Tehran, 19395-5746, Iran \\ Amir.Hashemi@cc.iut.ac.ir
}

\author{
Werner M. Seiler \\ Institut fur Mathematik, Universitat Kassel, \\ Heinrich-Plett-Straie 40, 34132 Kassel, \\ Germany \\ seiler@mathematik.uni-kassel.de
}

\begin{abstract}
We improve certain degree bounds for Gröbner bases of polynomial ideals in generic position. We work exclusively in deterministically verifiable and achievable generic positions of a combinatorial nature, namely either strongly stable position or quasi stable position. Furthermore, we exhibit new dimension- (and depth-)dependent upper bounds for the Castelnuovo-Mumford regularity and the degrees of the elements of the reduced Gröbner basis (w.r.t. the degree reverse lexicographical ordering) of a homogeneous ideal in these positions.
\end{abstract}

\section{Categories and Subject Descriptors}

F.2.2 [Analysis of Algorithms and Problem Complexity]: Nonnumerical Algorithms and Problems

\section{Keywords}

Polynomial ideals, Gröbner bases, Pommaret bases, generic positions, stability, degree, dimension, depth, CastelnuovoMumford regularity.

\section{INTRODUCTION}

Gröbner bases, introduced by Bruno Buchberger in his Ph.D. thesis (see e.g. 6, 7]), have become a powerful tool for constructive problems in polynomial ideal theory and related domains. For practical applications, in particular, the implementation in computer algebra systems, it is important to establish upper bounds for the complexity of determining a Gröbner basis for a given homogeneous polynomial ideal. Using Lazard's algorithm 23, a good measure to estimate such a bound, is an upper bound for the degree of the intermediate polynomials during the Gröbner basis computation. If the input ideal is not homogeneous, the maximal degree of the output Gröbner basis is not sufficient for this estimation. On the other hand, Möller and Mora 30] showed that to discuss degree bounds for Gröbner bases, one can restrict

Permission to make digital or hard copies of all or part of this work for personal or classroom use is granted without fee provided that copies are not made or distributed for profit or commercial advantage and that copies bear this notice and the full citation on the first page. To copy otherwise, to republish, to post on servers or to redistribute to lists, requires prior specific permission and/or a fee.

ISSAC'17, July 25-28, 2017, Kaiserslautern, Germany.

Copyright 2012 ACM X-XXXXX-XX-X/XX/XX ...\$5.00. to homogeneous ideals. Thus upper bounds for the degrees of the elements of Gröbner bases of homogeneous ideals, allow us to estimate the complexity of computing Gröbner bases in general.

Let us review some of the existing results in this direction. Let $\mathcal{P}$ be the polynomial ring $\mathbb{k}\left[x_{1}, \ldots, x_{n}\right]$ where $\mathbb{k}$ is of characteristic zero and $\mathcal{I} \subset \mathcal{P}$ be an ideal generated by homogeneous polynomials of degree at most $d$ with $\operatorname{dim}(\mathcal{I})=D$. The first doubly exponential upper bounds were proven by Bayer, Möller, Mora and Giusti, see 31, Chapter 38] for a comprehensive review of this topic. Based on results due to Bayer 2] and Galligo 14, 15, Möller and Mora 30 provided the upper bound $(2 d)^{(2 n+2)^{n+1}}$ for any Gröbner basis of $\mathcal{I}$. They also proved that this doubly exponential behavior cannot be improved. Simultaneously, Giusti [16] showed the upper bound $(2 d)^{2^{n-1}}$ for the degree of the reduced Gröbner basis (w.r.t. the degree reverse lexicographic order) of $\mathcal{I}$ when the ideal is in generic position. Then, using a self-contained and constructive combinatorial argument, Dubé 10 proved the so far sharpest degree bound $2\left(d^{2} / 2+d\right)^{2^{n-1}} \sim 2 d^{2^{n}}$.

In 2005, Caviglia and Sbarra 8] studied upper bounds for the Castelnuovo-Mumford regularity of homogeneous ideals. Analyzing Giusti's proof, they gave a simple proof of the upper bound $(2 d)^{2^{n-2}}$ for the degree reverse lexicographic Gröbner basis of an ideal $\mathcal{I}$ in generic position (they also showed that this bound holds independent of the characteristic of $\mathbb{k}$ ). Finally, Mayr and Ritscher 29], by following the tracks of Dubé 10, obtained the dimension-dependent upper bound $2\left(1 / 2 d^{n-D}+d\right)^{2^{D-1}}$ for any reduced Gröbner basis of $\mathcal{I}$. It is worth while remarking that there are also lower bounds for the complexity: $d^{2^{m}}$ with $m=n / 10-O(1)$ from the work of Mayr and Meyer 28] and $d^{2^{m}}$ where $m \sim n / 2$ due to Yap 37.

In this article, we will first improve Giusti's bound by showing that if $\mathcal{I}$ is in strongly stable position and $D>1$, then $2 d^{(n-D) 2^{D-1}}$ is a simultaneous upper bound for the Castelnuovo-Mumford regularity of $\mathcal{I}$ and for the maximal degree of the elements of the Gröbner basis of $\mathcal{I}$ (with respect to the degree reverse lexicographic order). Furthermore, we will sharpen the bound of Caviglia-Sbarra to $\left(d^{n-D}+\right.$ $(n-D)(d-1))^{2^{D-1}}$. We will see that neither of these bounds is always greater than the other. Finally, we will show that, if $\mathcal{I}$ is in quasi stable position and $D \leq 1$, Giusti's bound may be replaced by $n d-n+1$ (this result was already obtained by Lazard [23] when the ideal is in generic position). 
In the recent work 21], we showed how many variants of stable positions - including quasi stable and strongly stable position - can be achieved via linear coordinate transformations constructed with a deterministic algorithm.

The article is organized as follows. In the next section, we give basic notations and definitions. In Sections 4,5 and 6 we improve the degree bounds provided by Giusti, CavigliaSbarra and Lazard, respectively.

\section{PRELIMINARIES}

Throughout this article, we keep the following notations. Let $\mathcal{P}=\mathbb{k}\left[x_{1}, \ldots, x_{n}\right]$ be the polynomial ring (where $\mathbb{k}$ is of characteristic zero). A power product of the variables $x_{1}, \ldots, x_{n}$ is called term and $\mathbb{T}$ denotes the monoid of all terms in $\mathcal{P}$. We consider non-zero homogeneous polynomials $f_{1}, \ldots, f_{k} \in \mathcal{P}$ and the ideal $\mathcal{I}=\left\langle f_{1}, \ldots, f_{k}\right\rangle$ generated by them. We assume that $f_{i}$ is of degree $d_{i}$ and that the numbering is such that $d_{1} \geq d_{2} \geq \cdots \geq d_{k}>0$. We also set $d=d_{1}$. Furthermore, we denote by $\mathcal{R}=\mathcal{P} / \mathcal{I}$ the corresponding factor ring and by $D$ its dimension. Finally, we use throughout the degree reverse lexicographic order with $x_{n} \prec \cdots \prec x_{1}$.

The leading term of a polynomial $f \in \mathcal{P}$, denoted by $\mathrm{LT}(f)$, is the greatest term (with respect to $\prec$ ) appearing in $f$ and its coefficient is the leading coefficient of $f$ and we denote it by $\mathrm{LC}(f)$. The leading monomial of $f$ is the product $\operatorname{LM}(f)=\mathrm{LC}(f) \operatorname{LT}(f)$. The leading ideal of $\mathcal{I}$ is defined as $\operatorname{LT}(\mathcal{I})=\langle\operatorname{LT}(f) \mid f \in \mathcal{I}\rangle$. For the finite set $F=\left\{f_{1}, \ldots, f_{k}\right\} \subset \mathcal{P}, \operatorname{LT}(F)$ denotes the set $\left\{\operatorname{LT}\left(f_{1}\right), \ldots, \operatorname{LT}\left(f_{k}\right)\right\} . \quad$ A finite subset $G \subset \mathcal{I}$ is called a Gröbner basis of $\mathcal{I}$ w.r.t. $\prec$, if $\operatorname{LT}(\mathcal{I})=\langle\operatorname{LT}(G)\rangle$. We refer to [1] for more details on Gröbner bases.

Given a graded $\mathcal{P}$-module $X$ and a positive integer $s$, we denote by $X_{s}$ the set of all homogeneous elements of $X$ of degree $s$. To define the Hilbert regularity of an ideal, recall that the Hilbert function of $\mathcal{I}$ is defined by $\operatorname{HF}_{\mathcal{I}}(t)=$ $\operatorname{dim}_{\mathbb{k}}\left(\mathcal{R}_{t}\right)$; the dimension of $\mathcal{R}_{t}$ as a $\mathbb{k}$-linear space. From a certain degree on, this function of $t$ is equal to a polynomial in $t$, called Hilbert polynomial, and denoted by $\mathrm{HP}_{\mathcal{I}}$ (see 9] for more details on this topic). The Hilbert regularity of $\mathcal{I}$ is $\operatorname{hilb}(\mathcal{I})=\min \left\{m \mid \forall t \geq m, \operatorname{HF}_{\mathcal{I}}(t)=\operatorname{HP}_{\mathcal{I}}(t)\right\}$. Finally, recall that the Hilbert series of $\mathcal{I}$ is the power series $\operatorname{HS}_{\mathcal{I}}(t)=\sum_{s=0}^{\infty} \operatorname{HF}_{\mathcal{I}}(s) t^{s}$.

Proposition 2.1. There exists a univariate polynomial $p(t)$ with $p(1) \neq 0$ such that $\operatorname{HS}_{\mathcal{I}}(t)=p(t) /(1-t)^{D}$. Furthermore, $\operatorname{hilb}(\mathcal{I})=\max \{0, \operatorname{deg}(p)-d+1\}$.

For a proof of this result, we refer to [13, Thm. 7, page 130]. It follows immediately from Macaulay's theorem that the Hilbert function of $\mathcal{I}$ is the same as that of $\operatorname{LT}(\mathcal{I})$ and this provides an effective method to compute it using Gröbner bases, see e.g. [18].

Let us state some auxiliary results on regular sequences. Recall that a sequence of polynomials $f_{1}, \ldots, f_{k} \in \mathcal{P}$ is called regular if $f_{i}$ is a non-zero divisor on the ring $\mathcal{P} /\left\langle f_{1}, \ldots, f_{i-1}\right\rangle$ for $i=2, \ldots, k$. This is equivalent to the condition that $f_{i}$ does not belong to any associated prime of $\left\langle f_{1}, \ldots, f_{i-1}\right\rangle$. It can be shown that the Hilbert series of a regular sequence $f_{1}, \ldots, f_{k}$ is equal to $\prod_{i=1}^{k}\left(1-t^{d_{i}}\right) /\left(1-t^{n}\right)$, see e.g. 25]. The converse of this result is also true, see [13. Exercise 7, page 137]. In addition, these conditions are equivalent to the statement that $D=n-k$.
Lemma 2.2. ([25, Prop. 4.1, page 108]) There exist homogeneous polynomials $g_{1}, \ldots, g_{n-D} \in \mathcal{P}$ such that the following conditions hold:

(1) $\operatorname{deg}\left(g_{i}\right)=d_{i}$ for each $i$,

(2) $g_{i} \equiv \lambda_{i} f_{i} \bmod \left\langle f_{i+1}, \ldots, f_{k}\right\rangle$ for some $0 \neq \lambda_{i} \in \mathbb{k}$ for $i=1, \ldots, n-D$,

(3) $g_{1}, \ldots, g_{n-D}$ is regular sequence in $\mathcal{P}$.

DEFINITION 2.3. The depth of the homogeneous ideal $\mathcal{I}$ is defined as the maximal integer $\lambda$ such that there exists a regular sequence of linear forms $y_{1}, \ldots, y_{\lambda}$ on $\mathcal{P} / \mathcal{I}$.

Definition 2.4. The homogeneous ideal $\mathcal{I}$ is $m$-regular, if its minimal graded free resolution is of the form

$$
\begin{aligned}
0 \longrightarrow \bigoplus_{j} \mathcal{P}\left(e_{r j}\right) \longrightarrow \cdots \\
\quad \cdots \longrightarrow \bigoplus_{j} \mathcal{P}\left(e_{1 j}\right) \longrightarrow \bigoplus_{j} \mathcal{P}\left(e_{0 j}\right) \longrightarrow \mathcal{I} \longrightarrow 0
\end{aligned}
$$

with $e_{i j}-i \leq m$ for each $i, j$. The Castelnuovo-Mumford regularity of $\mathcal{I}$ is the smallest $m$ such that $\mathcal{I}$ is $m$-regular; we denote it by $\operatorname{reg}(\mathcal{I})$.

For more details on the regularity, we refer to $32,12,3$, 5]. It is well-known that in generic coordinates $\operatorname{reg}(\mathcal{I})$ is an upper bound for the degree of the Gröbner basis w.r.t. the degree reverse lexicographic order. This upper bound is sharp, if the characteristic of $\mathbb{k}$ is zero (see 3 ). A good measure to estimate the complexity of the computation of the Gröbner basis of $\mathcal{I}$ is the maximal degree of the polynomials which appear in this computation (see [22, 23, 16]).

Definition 2.5. We denote by $\operatorname{deg}(\mathcal{I}, \prec)$ the maximal degree of the elements of the reduced Gröbner basis of the nonzero homogeneous ideal $\mathcal{I}$ w.r.t. the term order $\prec$.

Theorem 2.6. ([25, Prop. 4.8, page 117]) If $\mathcal{I}$ is zerodimensional, then $\operatorname{deg}(\mathcal{I}, \prec) \leq d_{1}+\cdots+d_{n}-n+1$.

We conclude this section with a brief review of the theory of Pommaret bases. Suppose that $f \in \mathcal{P}$ and $\operatorname{LT}(f)=x^{\alpha}$ with $\alpha=\left(\alpha_{1}, \ldots, \alpha_{n}\right)$. We call $\max \left\{i \mid \alpha_{i} \neq 0\right\}$ the class of $f$, denoted by $\operatorname{cls}(f)$. Then the multiplicative variables of $f$ are $\mathcal{X}_{P}(f)=\left\{x_{\operatorname{cls}(f)}, \ldots, x_{n}\right\}$. Furthermore, $x^{\beta}$ is a Pommaret divisor of $x^{\alpha}$, written $\left.x^{\beta}\right|_{P} x^{\alpha}$, if $x^{\beta} \mid x^{\alpha}$ and $x^{\alpha-\beta} \in \mathbb{k}\left[x_{\operatorname{cls}(f)}, \ldots, x_{n}\right]$.

Definition 2.7. Let $\mathcal{H} \subset \mathcal{I}$ be a finite set such that no leading term of an element of $\mathcal{H}$ is a Pommaret divisor of the leading term of another element. Then $\mathcal{H}$ is called a Pommaret basis of $\mathcal{I}$ for $\prec$, if

$$
\mathcal{I}=\bigoplus_{h \in \mathcal{H}} \mathbb{k}\left[\mathcal{X}_{P}(h)\right] \cdot h
$$

One can easily show that any Pommaret basis is a (generally non-reduced) Gröbner basis of the ideal it generates. The main difference between Gröbner and Pommaret bases consists of the fact that by (1) any polynomial $f \in \mathcal{I}$ has a unique involutive standard representation. If an ideal $\mathcal{I}$ possesses a Pommaret basis $\mathcal{H}$, then $\operatorname{reg}(\mathcal{I})$ equals the maximal degree of an element of $\mathcal{H}$, cf. [34, Thm. 9.2]. The main 
drawback of Pommaret bases is however that they do not always exist. Indeed, a given ideal possesses a finite Pommaret basis, if and only if the ideal is in quasi stable position - see 34, Prop 4.4].

Definition 2.8. A monomial ideal $\mathcal{J}$ in $\mathcal{P}$ is called quasi stable, if for any term $m \in \mathcal{J}$ and all integers $i, j, s$ with $1 \leq j<i \leq n$ and $s>0$ such that $x_{i}^{s} \mid m$, there exists an exponent $t \geq 0$ such that $x_{j}^{t} m / x_{i}^{s} \in \mathcal{J}$. A homogeneous ideal $\mathcal{I}$ is in quasi stable position, if $\operatorname{LT}(\mathcal{I})$ is quasi stable.

In the sequel, we will use the following notations: given an ideal $\mathcal{I}$ in quasi stable position, we write $\mathcal{H}=\left\{h_{1}, \ldots, h_{s}\right\}$ for its Pommaret basis. Furthermore, for each $i$ we set $m_{i}=$ $\operatorname{LT}\left(h_{i}\right)$ and it is then easy to see that $\left\{m_{1}, \ldots, m_{s}\right\}$ forms a Pommaret basis of $\operatorname{LT}(\mathcal{I})$.

REMARK 2.9. Since any linear change of variables is a $\mathbb{k}$-linear automorphism of $\mathcal{P}$ preserving the degree, it follows trivially that the dimensions over $\mathbb{k}$ of the homogeneous components of a homogeneous ideal $\mathcal{I}$ or of its factor ring $\mathcal{R}$ remain invariant. Hence the Hilbert function and therefore also the Hilbert series, the Hilbert polynomial and the Hilbert regularity of $\mathcal{I}$ do not change. The same is obviously true for the Castelnuovo-Mumford regularity. In addition, due to the special form of the Hilbert series of the ideal generated by a regular sequence, we conclude that any regular sequence remains regular after a linear change of variables and hence the depth is invariant, too. Finally, we note that almost all linear changes of variables transform a given homogeneous ideal into quasi stable position (which is thus a generic position) [34]. It follows that to study any of the mentioned invariants of $\mathcal{I}$, w.l.o.g. we may assume that $\mathcal{I}$ is in quasi stable position.

\section{IMPROVING GIUSTI'S UPPER BOUND}

In 1984, Giusti 16 established the upper bound $(2 d)^{2^{n-1}}$ for $\operatorname{deg}(\mathcal{I}, \prec)$ in the case that the coordinates are in generic position. The key point of Giusti's proof is the use of the combinatorial structure of the generic initial ideal in characteristic zero. Later on, Mora [31, Ch. 38], by a deeper analysis of Giusti's proof, improved this bound to $(d+1)^{(n-D) 2^{D-\lambda}}$ where $\lambda$ is the depth of $\mathcal{I}$. In this section, we improve Mora's bound by following his general approach and correcting some flaws in his method. Our presentation seems to be simpler than the ones by Mora and Giusti.

We first note that for a given ideal in quasi stable position, we are able to reduce the number of variables by the depth of the ideal to obtain a sharper bound for $\operatorname{deg}(\mathcal{I}, \prec)$. A novel proof $\grave{a}$ la Pommaret of this result is given below.

Proposition 3.1. Suppose that $U(n, d, D)$ is a function depending in $n, d$ and $D$ so that $\operatorname{deg}(\mathcal{I}, \prec) \leq U(n, d, D)$ for any ideal $\mathcal{I}$ which is in quasi stable position and is generated by homogeneous polynomials of degree at most $d$ in $n$ variables. Then, $\operatorname{deg}(\mathcal{I}, \prec) \leq U(n-\lambda, d, D-\lambda)$ where $\operatorname{depth}(\mathcal{I})=\lambda$.

Proof. Let $t$ be the maximal class of the elements in $\mathcal{H}$. It is shown in [34, Prop 2.20] that in quasi-stable position the variables $x_{t+1}, \ldots, x_{n}$ define a regular sequence on $\mathcal{R}$ and that thus $\lambda=n-t$ (note that this reference distinguishes between $\operatorname{depth}(\mathcal{I})$ and $\operatorname{depth}(\mathcal{R})$ with the two related by
$\operatorname{depth}(\mathcal{R})=\operatorname{depth}(\mathcal{I})-1$; what we call here $\operatorname{depth}(\mathcal{I})$ corresponds to $\operatorname{depth}(\mathcal{R})$ in 34$])$. By definition of $t$, no leading term of an element of $\mathcal{H}$ is divisible by any of these variables. Thus $\tilde{\mathcal{H}}=\left.\mathcal{H}\right|_{x_{t+1}=\cdots=x_{n}=0}$ is the Pommaret basis of the ideal $\tilde{\mathcal{I}}=\left.\mathcal{I}\right|_{x_{t+1}=\cdots=x_{n}=0}$ in $\mathbb{k}\left[x_{1}, \ldots, x_{t}\right]$ and hence $\operatorname{deg}(\mathcal{I}, \prec)=\operatorname{deg}(\tilde{\mathcal{I}}, \prec)$. This entails our claim.

Corollary 3.2. As a similar statement to Prop. 3.1. suppose that $R(n, d, D)$ is a function depending in $n, d$ and $D$ such that $\operatorname{reg}(\mathcal{I}) \leq R(n, d, D)$. Then, $\operatorname{reg}(\mathcal{I}) \leq R(n-$ $\lambda, d, D-\lambda)$.

Proof. This claim follows by the same argument as in the proof of Prop. 3.1 and using the facts that for each $f$ in the Pommaret bases $\mathcal{H}$ the corresponding element $\tilde{f} \in \tilde{\mathcal{H}}$ has the same degree as $f$ and in quasi stable position $\operatorname{reg}(\mathcal{I})=$ $\operatorname{reg}(\tilde{\mathcal{I}})$ is given by the maximal degree of the elements of $\mathcal{H}$ and $\tilde{\mathcal{H}}$.

To state the refined version of Giusti's bound, we need to recall the crystallisation principle. Let $A=\left(a_{i j}\right) \in \mathrm{GL}(n, \mathbb{k})$ be an $n \times n$ invertible matrix. By $A . \mathcal{I}$ we mean the ideal generated by the polynomials $A$. $f$ with $f \in \mathcal{I}$ where $A . f=$ $f\left(\sum_{i=1}^{n} a_{i 1} x_{i}, \ldots, \sum_{i=1}^{n} a_{i n} x_{i}\right)$. The following fundamental theorem is due to Galligo [14].

THEOREM 3.3. There exists a non-empty Zariski open subset $\mathcal{U} \subset \mathrm{GL}(n, \mathbb{k})$ such that $\operatorname{LT}(A \cdot \mathcal{I})=\operatorname{LT}\left(A^{\prime} \cdot \mathcal{I}\right)$ for all matrices $A, A^{\prime} \in \mathcal{U}$.

Definition 3.4. The monomial ideal $\operatorname{LT}(A . \mathcal{I})$ with $A \in$ $\mathcal{U}$ and $\mathcal{U}$ as given in Theorem 3.3 is called the generic initial ideal of $\mathcal{I}$ (w.r.t. $\prec$ ) and is denoted by $\operatorname{gin}(\mathcal{I})$.

Suppose that $\mathcal{I}=\left\langle f_{1}, \ldots, f_{k}\right\rangle$ and that for some $s \in \mathbb{N}$ we have $\operatorname{deg}\left(f_{i}\right) \leq s$ for all $i$ and $\operatorname{gin}(\mathcal{I})$ has no minimal generator in degree $s+1$. Then, the crystallisation principle (CP) states that for each $m$ in the generating set of $\operatorname{gin}(\mathcal{I})$ we have $\operatorname{deg}(m) \leq s$, see [17, Prop 2.28]. Note that this principle holds only in characteristic zero and it has been proven only for generic initial ideals and for lexicographic ideals (see [17, Thm. 3.8]).

Giusti's proof consists in applying this property along with an induction on the number of variables. One crucial fact in this direction is that CP also holds for a generic initial ideal modulo the last variable. Below, we will show that both properties remain true for arbitrary strongly stable ideals.

Definition 3.5. A monomial ideal $\mathcal{J}$ is called strongly stable, if for any term $m \in \mathcal{J}$ we have $x_{j} m / x_{i} \in \mathcal{J}$ for all $i$ and $j$ such that $j<i$ and $x_{i}$ divides $m$. A homogeneous ideal $\mathcal{I}$ is in strongly stable position, if $\operatorname{LT}(\mathcal{I})$ is strongly stable.

Proposition 3.6. Let $\mathcal{I}$ be in strongly stable position. Then, CP holds for $\operatorname{LT}(\mathcal{I})$.

Proof. The following arguments are inspired by [31, page 728]. Let us consider an integer $s \geq d$. Suppose that we are computing a Gröbner basis of $\mathcal{I}$ using Buchberger's algorithm and by applying the normal strategy. In addition, assume that we have already computed the set $G=\left\{g_{1}, \ldots g_{t}\right\}$ up to degree $s$ (this set will be enlarged to a Gröbner basis of $\mathcal{I}$ ), and there is no new polynomial of degree $s+1$ to be 
added into $G$. Note that we have chosen $s \geq d$ to be sure that $G$ generates $\mathcal{I}$. To prove the assertion, it suffices to show that $G$ is a Göbner basis of $\mathcal{I}$.

We introduce the set $M_{s}=\langle\operatorname{LT}(G)\rangle_{s} \cap \mathbb{T}$. We now claim that for each pair of terms $x^{\alpha}=x_{1}^{\alpha_{1}} \cdots x_{n}^{\alpha_{n}} \neq x^{\beta}=$ $x_{1}^{\beta_{1}} \cdots x_{n}^{\beta_{n}}$ in it either $\operatorname{deg}\left(\operatorname{lcm}\left(x^{\alpha}, x^{\beta}\right)\right)=s+1$ or there exists a further term $x^{\gamma} \in M_{s} \backslash\left\{x^{\alpha}, x^{\beta}\right\}$ such that

- $x^{\gamma} \mid \operatorname{lcm}\left(x^{\alpha}, x^{\beta}\right)$,

- $\operatorname{deg}\left(\operatorname{lcm}\left(x^{\gamma}, x^{\alpha}\right)\right)<\operatorname{deg}\left(\operatorname{lcm}\left(x^{\alpha}, x^{\beta}\right)\right)$,

- $\operatorname{deg}\left(\operatorname{lcm}\left(x^{\gamma}, x^{\beta}\right)\right)<\operatorname{deg}\left(\operatorname{lcm}\left(x^{\alpha}, x^{\beta}\right)\right)$.

If this claim is true, then Buchberger's second criterion implies that it suffices to consider those pairs $\left\{g_{i}, g_{j}\right\}$ with $\operatorname{deg}\left(\operatorname{lcm}\left(\operatorname{LT}\left(g_{i}\right), \operatorname{LT}\left(g_{j}\right)\right)\right)=s+1$. If for each such pair the corresponding S-polynomial reduces to zero, then $G$ is a Gröbner basis and we are done. Otherwise, there exists a new generator of degree $s+1$ contradicting the made assumptions.

For proving the made claim, it suffices to show that, if $\operatorname{deg}\left(\operatorname{lcm}\left(x^{\alpha}, x^{\beta}\right)\right)>s+1$, then there exists a term $x^{\gamma} \in$ $M_{s} \backslash\left\{x^{\alpha}, x^{\beta}\right\}$ satisfying the above conditions. Let $j$ be an integer such that $\alpha_{j} \neq \beta_{j}$ and $\alpha_{j+1}=\beta_{j+1}, \ldots, \alpha_{n}=\beta_{n}$. W.l.o.g., we may assume that $\alpha_{j}>\beta_{j}$. Since $x^{\alpha}$ and $x^{\beta}$ have the same degree, there is an index $i<j$ such that $\beta_{i}>\alpha_{i}$. The strongly stable position of $\mathcal{I}$ implies that $M_{s}$ is a strongly stable set. Therefore the term $x^{\gamma}=x_{i} x^{\alpha} / x_{j}$ satisfies $x^{\gamma} \in M_{s} \backslash\left\{x^{\alpha}, x^{\beta}\right\}$ and $x^{\gamma} \mid \operatorname{lcm}\left(x^{\alpha}, x^{\beta}\right)$. Furthermore, $\operatorname{deg}\left(\operatorname{lcm}\left(x^{\gamma}, x^{\alpha}\right)\right)=s+1<\operatorname{deg}\left(\operatorname{lcm}\left(x^{\alpha}, x^{\beta}\right)\right)$ and $\operatorname{deg}\left(\operatorname{lcm}\left(x^{\gamma}, x^{\beta}\right)\right)=\operatorname{deg}\left(\operatorname{lcm}\left(x^{\alpha}, x^{\beta}\right)\right)-1$.

EXAMPLE 3.7. One should note that strong stability of the leading term ideal does not imply that it is the generic initial ideal, as the following example due to Green [17] shows: $\mathcal{I}=$ $\left\langle x_{1} x_{3}, x_{1} x_{2}+x_{2}^{2}, x_{1}^{2}\right\rangle \subset \mathbb{k}\left[x_{1}, x_{2}, x_{3}\right]$. Its leading term ideal $\mathrm{LT}(\mathcal{I})=\left\langle x_{1} x_{3}, x_{1} x_{2}, x_{1}^{2}, x_{2}^{2} x_{3}, x_{2}^{3}\right\rangle$ is strongly stable, but we find $\operatorname{gin}(\mathcal{I})=\left\langle x_{2}^{2}, x_{1} x_{2}, x_{1}^{2}, x_{1} x_{3}^{2}\right\rangle \neq \mathrm{LT}(\mathcal{I})$. Nevertheless, it is clear that both $\operatorname{LT}(\mathcal{I})$ and $\operatorname{gin}(\mathcal{I})$ satisfy $C P$.

As a consequence of the proof of this proposition, we can infer a generalization of $\mathrm{CP}$.

COROLlary 3.8. Suppose we know in advance that $\mathcal{I}$ is in strongly stable position. Let us fix an integer $t$ (not necessarily greater than d). Suppose that we are computing a Gröbner basis for $\mathcal{I}$ using Buchberger's algorithm and applying the normal strategy. Assume that we have treated all S-polynomials of degree at most $t$ and $G_{t}$ is the set of all polynomials computed so far. If all S-polynomials of degree $t+1$ reduce to zero, then any critical pair $\{f, g\}$ with $\max \{\operatorname{deg}(f), \operatorname{deg}(g)\} \leq t$ is superfluous. In particular, $G_{t}$ is a Gröbner basis for $\left\langle\overline{\mathcal{I}}_{\leq t}\right\rangle$.

In the sequel, for an index $i$ we denote by $\mathcal{I}_{i}$ the ideal $\left.\mathcal{I}\right|_{x_{i}=\cdots=x_{n}=0} \subset \mathbb{k}\left[x_{1}, \ldots, x_{i-1}\right]$. Since we assume that $\prec$ is the degree reverse lexicographic term order, strongly stable position of $\mathcal{I}$ entails that $\mathcal{I}_{i}$ is in strongly stable position, too, for any index $i$. The essence of Giusti's approach consists of finding, by repeated evaluation, relations between $\operatorname{deg}(\mathcal{I}, \prec)$ and $\operatorname{deg}\left(\mathcal{I}_{i}, \prec\right)$ for $i=n, \ldots, n-D+1$. For this purpose, we introduce some further notations for an ideal $\mathcal{I}$ in strongly stable position. We denote by $N(\mathcal{I})$ the set of all terms $m \notin \operatorname{LT}(\mathcal{I})$. If $\operatorname{dim}(\mathcal{I})=0$, then we define
$F(\mathcal{I})=N(\mathcal{I})$. Otherwise we set $F(\mathcal{I})=\left\{\tau x_{n}^{a} \in N(\mathcal{I}) \mid \tau \in\right.$ $F\left(\mathcal{I}_{n}\right)$ and $\left.\operatorname{deg}\left(\tau x_{n}^{a}\right)<\operatorname{deg}(\mathcal{I}, \prec)\right\}$. Since $\mathcal{I}$ is in strongly stable position, $N(\mathcal{I})$ is strongly stable for the reverse ordering of the variables. More precisely, if $x^{\alpha} \in N(\mathcal{I})$ with $\alpha_{i}>0$, then we claim that $x_{j} x^{\alpha} / x_{i} \in N(\mathcal{I})$ for any $j>i$. Indeed, otherwise it belonged to $\operatorname{LT}(\mathcal{I})$ and thus - since $\mathrm{LT}(\mathcal{I})$ is strongly stable $-x^{\alpha} \in \mathrm{LT}(\mathcal{I})$ which is a contradiction.

Lemma 3.9. Suppose that $\mathcal{I}$ is in strongly stable position. Then the following statements hold.

(a) $\operatorname{deg}(\mathcal{I}, \prec) \leq \max \left\{d, \operatorname{deg}\left(\mathcal{I}_{n}, \prec\right)\right\}+\# F\left(\mathcal{I}_{n}\right)$,

(b) $\# F(\mathcal{I}) \leq\left(\max \left\{d, \# F\left(\mathcal{I}_{n}\right)\right\}\right)^{2}$.

(Here \#X denotes the cardinality of a finite set $X$.)

Proof. (a) Let $G$ be the reduced Gröbner basis of $\mathcal{I}$ for $\prec$. Because of our use of the degree reverse lexicographic term order, we easily see that $\left.G\right|_{x_{n}=0}$ is the reduced Gröbner basis of $\mathcal{I}_{n}$ for $\prec$. Let $G^{\prime} \subset G$ be the subset of all polynomials in $G$ of maximal degree. We distinguish two cases. If $\operatorname{LT}\left(G^{\prime}\right) \cap \mathbb{k}\left[x_{1}, \ldots, x_{n-1}\right] \neq \emptyset$, then obviously $\operatorname{deg}(\mathcal{I}, \prec)=\operatorname{deg}\left(\mathcal{I}_{n}, \prec\right)$ and the assertion is proved.

Otherwise, CP (applicable by Prop. 3.6) implies that for each degree $\max \left\{d, \operatorname{deg}\left(\mathcal{I}_{n}, \prec\right)\right\}<i \leq \operatorname{deg}(\mathcal{I}, \prec)$ there exists a polynomial $g_{i} \in G$ with $\operatorname{deg}\left(g_{i}\right)=i$ (note that if $\operatorname{deg}(\mathcal{I}, \prec)=d$ then $(a)$ holds and we are done). Thus, we can write $\operatorname{LT}\left(g_{i}\right)$ in the form $x_{n}^{a_{i}} \tau_{i}$ with $a_{i}>0$ and $\tau_{i} \in \mathbb{k}\left[x_{1}, \ldots, x_{n-1}\right]$. We claim that $\tau_{i} \in F\left(\mathcal{I}_{n}\right)$. Writing $\tau_{i}=x_{i_{1}}^{\alpha_{i_{1}}} \cdots x_{i_{k}}^{\alpha_{i_{k}}}$ where $i_{1}<\cdots<i_{k}$, we may conclude by the assumed reducedness of $G$ that $\tau_{i} \notin \mathrm{LT}(\mathcal{I})$ and by the strong stability of $\operatorname{LT}(\mathcal{I})$ that $x_{i_{1}}^{\alpha_{i_{1}}} \cdots x_{i_{k}}^{\alpha_{i_{k}}+a_{i}} \in$ $\operatorname{LT}(\mathcal{I})$. Hence there exists an integer $a>0$ such that $x_{i_{1}}^{\alpha_{i_{1}}} \cdots x_{i_{k}}^{\alpha_{i_{k}}+a-1} \notin \operatorname{LT}(\mathcal{I})$ and $x_{i_{1}}^{\alpha_{i_{1}}} \cdots x_{i_{k}}^{\alpha_{i_{k}}+a} \in \operatorname{LT}(\mathcal{I})$. It follows that there exists a generator $g \in G \cap \mathcal{I}_{n}$ such that its leading term $\operatorname{LT}(g)=x_{i_{1}}^{\beta_{i_{1}}} \cdots x_{i_{k}}^{\beta_{i_{k}}}$ divides the latter term. We must have $\beta_{\ell} \leq \alpha_{\ell}$ for each $\ell<i_{k}$ and $\beta_{i_{k}}=\alpha_{i_{k}}+a$ by definition of $\bar{a}$. Furthermore, the strong stability of $\operatorname{LT}(\mathcal{I})$ implies that $\operatorname{deg}(g)>\operatorname{deg}\left(\tau_{i}\right)$, as otherwise another generator $g^{\prime} \in G$ existed with $\operatorname{LT}\left(g^{\prime}\right) \mid \tau_{i}$. Thus $\operatorname{deg}\left(\tau_{i}\right)<\operatorname{deg}\left(\mathcal{I}_{n}, \prec\right)$. If we write $\tau_{i}=\bar{\tau}_{i} x_{i_{k}}^{\alpha_{i_{k}}}$, then there only remains to show that $\bar{\tau}_{i} \in F\left(\mathcal{I}_{i_{k}}\right)$, as $\tau_{i} \in N\left(\mathcal{I}_{n}\right)$ is a trivial consequence of $\tau_{i} \in N(\mathcal{I})$. If $\operatorname{dim}\left(\mathcal{I}_{i_{k}}\right)=0$, this follows immediately from $F\left(\mathcal{I}_{i_{k}}\right)=N\left(\mathcal{I}_{i_{k}}\right)$. Otherwise we repeated the same arguments as above.

Thus for each $i$ with $\max \left\{d, \operatorname{deg}\left(\mathcal{I}_{n}, \prec\right)\right\}<i \leq \operatorname{deg}(\mathcal{I}, \prec)$ there exists a generator $g_{i} \in G$ such that $\operatorname{LT}\left(g_{i}\right)=x_{n}^{a_{i}} \tau_{i}$ and $\tau_{i} \in F\left(\mathcal{I}_{n}\right)$. Since $G$ is reduced, the terms $\tau_{i}$ are pairwise different. Hence $\operatorname{deg}(\mathcal{I}, \prec)-\max \left\{d, \operatorname{deg}\left(\mathcal{I}_{n}, \prec\right)\right\} \leq \# F\left(\mathcal{I}_{n}\right)$ and this proves $(a)$.

To show $(b)$, we introduce for each degree $\delta \in \mathbb{N}$ the subset $F_{\delta}(\mathcal{I})=\left\{x_{n}^{\delta} \tau \mid x_{n}^{\delta} \tau \in F(\mathcal{I})\right\}$. By definition, $x_{n}^{\delta} \tau \in F_{\delta}(\mathcal{I})$ implies $\tau \in F\left(\mathcal{I}_{n}\right)$ and thus $\# F_{\delta}(\mathcal{I}) \leq \# F\left(\mathcal{I}_{n}\right)$. Since we used in the proof of $(a) \mathrm{CP}$, the claims proven there are true only for polynomials of degree at least $d$. Thus in the sequel we shall replace $\# F\left(\mathcal{I}_{n}\right)$ by $\max \left\{d, \# F\left(\mathcal{I}_{n}\right)\right\}$. We observe that the maximal $\delta$ such that $x_{n}^{\delta} \tau \in F(\mathcal{I})$ is $\max \left\{d, \# F\left(\mathcal{I}_{n}\right)\right\}$ and thus

$$
\# F(\mathcal{I}) \leq \sum_{\delta=0}^{\max \left\{d, \# F\left(\mathcal{I}_{n}\right)\right\}-1} \max \left\{d, \# F\left(\mathcal{I}_{n}\right)\right\}
$$

which immediately yields the inequality in $(b)$. 
REMARK 3.10. Mora [31, Thm. 38.2.7] presented another version of this lemma. Instead of our set $F(\mathcal{I})$, he defined $\tilde{F}(\mathcal{I})=\left\{\tau x_{n}^{a} \in N(\mathcal{I}) \mid \tau \in N\left(\mathcal{I}_{n}\right), \operatorname{deg}\left(\tau x_{n}^{a}\right)<\operatorname{deg}(\mathcal{I}, \prec)\right\}$ which differs only in the condition on $\tau$. Assuming the equality $\tilde{F}_{0}(\mathcal{I})=\tilde{F}\left(\mathcal{I}_{n}\right)$ where $\tilde{F}_{0}(\mathcal{I})$ contains the elements of $\tilde{F}(\mathcal{I})$ with $a=0$, he proved the following two properties:

(a) $\operatorname{deg}(\mathcal{I}, \prec) \leq \operatorname{deg}\left(\mathcal{I}_{n}, \prec\right)+\# \tilde{F}\left(\mathcal{I}_{n}\right)$,

(b) $\# \tilde{F}(\mathcal{I}) \leq\left(\# \tilde{F}\left(\mathcal{I}_{n}\right)\right)^{2}$.

However, in general these assertions are not correct - not even for an ideal in generic position. Indeed, in general we have only $\tilde{F}\left(\mathcal{I}_{n}\right) \subseteq \tilde{F}_{0}(\mathcal{I})$ and if $\operatorname{dim}(\mathcal{I})>0$ and $\operatorname{deg}\left(\mathcal{I}_{1} \prec\right)<$ $\operatorname{deg}(\mathcal{I}, \prec)$ then equality does not hold. As a concrete example consider $\mathcal{I}=\left\langle x_{1}^{2}, x_{2}^{11} x_{1}\right\rangle \subset \mathbb{k}\left[x_{1}, x_{2}\right]$. We perform a generic linear change $x_{1}=a y_{1}+b y_{2}$ and $x_{2}=c y_{1}+$ $d y_{2}$ with parameters $a, b, c, d \in \mathbb{k}$. The leading term ideal of the new ideal is then $\left\langle y_{1}^{2}, y_{2}^{11} y_{1}\right\rangle$. This show that $\mathcal{I}=$ $\operatorname{gin}(\mathcal{I})$ and therefore the original coordinates for $\mathcal{I}$ are already generic. We have $\mathcal{I}_{2}=\left\langle x_{1}^{2}\right\rangle, F\left(\mathcal{I}_{2}\right)=\left\{1, x_{1}\right\}$ and $\operatorname{deg}\left(\mathcal{I}_{2}, \prec\right)=2$. Furthermore, we have $\tilde{F}(\mathcal{I})=\left\{x_{2}^{11}\right\} \cup$ $\left\{x_{2}^{i}, x_{2}^{i} x_{1} \mid i=0, \ldots, 10\right\}$ and $\# \tilde{F}(\mathcal{I})=23$. Thus, $12=$ $\operatorname{deg}(\mathcal{I}, \prec) \not \mathbb{d e g}\left(\mathcal{I}_{n}, \prec\right)+\# \tilde{F}\left(\mathcal{I}_{n}\right)=2+2=4$ and $23=$ $\# \tilde{F}(\mathcal{I}) \not \subset\left(\# \tilde{F}\left(\mathcal{I}_{n}\right)\right)^{2}=4$.

In the case that $\mathcal{I}$ is a zero-dimensional ideal, we can derive explicit upper bounds for $\operatorname{deg}(\mathcal{I}, \prec)$ and $\# F(\mathcal{I})$ using the following well-known lemma. We include an elementary proof for the sake of completeness.

Lemma 3.11. Let $\mathcal{I}$ be a zero-dimensional ideal. Then

(a) $\operatorname{deg}(\mathcal{I}, \prec) \leq d_{1}+\cdots+d_{n}-n+1$,

(b) $\# F(\mathcal{I}) \leq d_{1} \cdots d_{n}$

Proof. (a) was already proven in Thm. 2.6 We present now an elementary proof for $(b)$. The assumption $\operatorname{dim}(\mathcal{I})=$ 0 implies that $\# F(\mathcal{I})=\operatorname{dim}_{\mathbb{k}}(\mathcal{P} / \mathcal{I})$ and this dimension is equal to the sum of the coefficients of the Hilbert series of $\mathcal{I}$ (which is of course a polynomial here). We may assume w.l.o.g. that the first $n$ generators $f_{1}, \ldots, f_{n}$ form a regular sequence (Lem. 2.2). Thus the Hilbert series of $\mathcal{I}^{\prime}=\left\langle f_{1}, \ldots, f_{n}\right\rangle$ is $\operatorname{HS}_{\mathcal{I}^{\prime}}(t)=\prod_{i=1}^{n}\left(1+\cdots+t^{d_{i}-1}\right)$ and $\operatorname{dim}_{\mathbb{k}}\left(\mathcal{P} / \mathcal{I}^{\prime}\right)$ is at most $\operatorname{HS}_{\mathcal{I}^{\prime}}(1)=d_{1} \cdots d_{n}$. We obviously have $\operatorname{dim}_{\mathbb{k}}(\mathcal{P} / \mathcal{I}) \leq \operatorname{dim}_{\mathbb{k}}\left(\mathcal{P} / \mathcal{I}^{\prime}\right)$ and this proves the assertion.

We state now the main result of this section.

THEOREM 3.12. If the ideal $\mathcal{I}$ is in strongly stable position, then $\# F(\mathcal{I}) \leq d^{(n-D) 2^{D}}$ and

$$
\operatorname{deg}(\mathcal{I}, \prec) \leq \max \left\{(n-D+1)(d-1)+1,2 d^{(n-D) 2^{D-1}}\right\} .
$$

Proof. We proceed by induction over $D=\operatorname{dim}(\mathcal{I})$. In this proof without loss of generality, we may assume that $d \geq 2$. If $D=0$, the assertions follow immediately from Lem. 3.11 For $D>0$, we exploit that then $\operatorname{dim}(\mathcal{I})=$ $\operatorname{dim}\left(\mathcal{I}_{n}\right)+1$ and that we may consider $\mathcal{I}_{n}$ as an ideal in $\mathbb{k}\left[x_{1}, \ldots, x_{n-1}\right]$. Lem. 3.9 now entails that

$$
\begin{aligned}
\# F(\mathcal{I}) & \leq \max \left\{d, \# F\left(\mathcal{I}_{n}\right)\right\}^{2} \\
& \leq\left(d^{(n-1-(D-1)) 2^{D-1}}\right)^{2}=d^{(n-D) 2^{D}}
\end{aligned}
$$

and thus the first inequality.

For the second inequality, Thm. 5.3 which will be proven in the last section, provides the starting point for the induction, as it immediately implies our claim for $D \leq 1$. For $D \geq 2$, we obviously have $(n-1-(D-1)+1)(d-1)+1 \leq$ $d^{(n-D) 2^{D-1}}$ and $2 d^{(n-1-(D-1)) 2^{D-2}} \leq d^{(n-1-(D-1)) 2^{D-1}}$. We can thus rewrite the induction hypothesis as

$$
\begin{aligned}
& \operatorname{deg}\left(\mathcal{I}_{n}, \prec\right) \leq \max \{(n-1-(D-1)+1)(d-1)+1, \\
&\left.2 d^{(n-1-(D-1)) 2^{D-2}}\right\} \leq d^{(n-D) 2^{D-1}} .
\end{aligned}
$$

Again by Lem. 3.9, we can also estimate

$$
\begin{aligned}
\operatorname{deg}(\mathcal{I}, \prec) & \leq \max \left\{d, \operatorname{deg}\left(\mathcal{I}_{n}, \prec\right)\right\}+\# F\left(\mathcal{I}_{n}\right) \\
& \leq d^{(n-D) 2^{D-1}}+d^{(n-1-(D-1)) 2^{D-1}} \\
& =2 d^{(n-D) 2^{D-1}}
\end{aligned}
$$

proving the second assertion.

EXAMPLE 3.13. Let us consider the values $n=2, d=2$ and $D=0$. The above theorem states $\operatorname{deg}(\mathcal{I}, \prec) \leq 2^{2}=4$. Consider the ideal $\mathcal{I}=\left\langle x_{1}^{2}, x_{1} x_{2}+x_{2}^{2}\right\rangle$. By performing a generic linear change of coordinates, we get $\operatorname{gin}(\mathcal{I})=$ $\left\langle x_{2} x_{1}, x_{1}^{2}, x_{2}^{3}\right\rangle$. Therefore $\# F(\mathcal{I})=4 \leq 4$ and $\operatorname{deg}(\mathcal{I}, \prec)=$ $3 \leq 4$ confirming the accuracy of the presented upper bounds. It should be noted that for such a zero-dimensional ideal Theorem 2.6 provides the best upper bound for $\operatorname{deg}(\mathcal{I}, \prec)$, namely $d_{1}+\cdots+d_{n}-n+1$ which is equal to the exact value 3 for this example.

Using Prop. 3.1 we obtain even sharper bounds depending on both the dimension and the depth of $\mathcal{I}$. We continue to write $\operatorname{dim}(\mathcal{I})=D$ and $\operatorname{depth}(\mathcal{I})=\lambda$. It is well-known that we always have $D \geq \lambda$ (a simple proof using Pommaret bases can be found in 34 after Prop. 3.19). If $D=\lambda$, then $\mathcal{R}$ is Cohen-Macaulay. In this case, a nearly optimal upper bound for $\operatorname{deg}(\mathcal{I}, \prec)$ exists. Recall that a homogeneous ideal $\mathcal{I} \subset \mathcal{P}$ is in Nother position, if the ring extension $\mathbb{k}\left[x_{n-D+1}, \ldots, x_{n}\right] \hookrightarrow \mathcal{P} / \mathcal{I}$ is integral. Alternatively, Nother position can be defined combinatorially as a weakened version of quasi stable position (see [21, Thm. 4.4]).

Theorem 3.14. ([25, Prop. 4.8, page 117]) Let $\mathcal{R}$ be a Cohen-Macaulay ring with $\mathcal{I}$ in Nother position. Then, $\operatorname{deg}(\mathcal{I}, \prec) \leq d_{1}+\cdots+d_{n-D}-(n-D)+1$.

For the rest of this section, we thus assume that $\mathcal{R}$ is not Cohen-Macaulay, i. e. that $D>\lambda$.

COROLlary 3.15. If $\mathcal{I}$ is in strongly stable position and $D>1$, then $\# F(\mathcal{I}) \leq d^{(n-D) 2^{D-\lambda-1}}$ and $\operatorname{deg}(\mathcal{I}, \prec) \leq$ $2 d^{(n-D) 2^{D-\lambda-1}}$.

The maximal degree of an element of the Pommaret basis of an ideal in quasi stable position equals the CastelnuovoMumford regularity 34, Cor. 9.5]. If the ideal is even in stable position, then the Pommaret basis coincides with the reduced Gröbner basis [26, Thm. 2.15]. These considerations imply now immediately the following two results.

COROllary 3.16. If the ideal $\mathcal{I}$ is in strongly stable position and $D>1$, then $\operatorname{reg}(\mathcal{I}) \leq 2 d^{(n-D) 2^{D-\lambda-1}}$.

COROLlary 3.17. Let the ideal $\mathcal{I}$ be in quasi stable position, $\mathcal{H}$ its Pommaret basis and $D>1$. If we write $\operatorname{deg}(\mathcal{H})$ for the maximal degree of an element of $\mathcal{H}$, then $\operatorname{deg}(\mathcal{I}, \prec) \leq$ $\operatorname{deg}(\mathcal{H}) \leq 2 d^{(n-D) 2^{D-\lambda-1}}$. 


\section{IMPROVING THE UPPER BOUND OF CAVIGLIA-SBARRA}

In 2005, Caviglia and Sbarra 8] gave a simple proof for the upper bound $(2 d)^{2^{n-2}}$ for $\operatorname{deg}(\mathcal{I}, \prec)$ when the coordinates are in generic position by analyzing Giusti's proof and exploiting some properties of quasi stable ideals. We will now improve this bound to a dimension dependent bound. As a by-product, we will show that the notion of genericity that one needs here is strongly stable position.

We begin with a quick review of the approach of Caviglia and Sbarra [8. For any monomial ideal $\mathcal{J} \subset \mathcal{P}$ let $G(\mathcal{J})$ be its unique minimal generating set. We write $\operatorname{deg}_{i}(\mathcal{J})=$ $\max \left\{\operatorname{deg}_{i}(u) \mid u \in G(\mathcal{J})\right\}$ where $\operatorname{deg}_{i}$ denotes the degree in the variable $x_{i}$. Slightly changing our previous notation, we now denote by $\mathcal{J}_{i}$ the ideal $\left.\mathcal{J}\right|_{x_{i+1}=\cdots=x_{n}=0} \subset \mathbb{k}\left[x_{1}, \ldots, x_{i}\right]$. It follows immediately from the definition of a quasi stable ideal that $\operatorname{deg}_{i}\left(\mathcal{J}_{i}\right)=\operatorname{deg}_{i}(\mathcal{J})$. We note that two distinct terms in $G(\mathcal{J})$ must differ already in the first $n-1$ variables because of the minimality of $G(\mathcal{J})$. Hence $\# G(\mathcal{J}) \leq$ $\prod_{i=1}^{n-1}\left(\operatorname{deg}_{i}(\mathcal{J})+1\right)$.

Assume that $\mathcal{I}$ is in quasi stable position and $\mathcal{I}$ satisfies CP w.r.t. d. CP implies that $\operatorname{deg}(\mathcal{I}, \prec)-d+1 \leq \# G(\operatorname{LT}(\mathcal{I}))$ and hence $\operatorname{deg}(\mathcal{I}, \prec) \leq d-1+\prod_{i=1}^{n-1}\left(\operatorname{deg}_{i}(\operatorname{LT}(\overline{\mathcal{I}}))+1\right)$. Quasi stability of $\operatorname{LT}(\mathcal{I})$ implies that $\operatorname{deg}_{i}(\operatorname{LT}(\mathcal{I}))=\operatorname{deg}\left(\mathcal{I}_{i}, \prec\right)$ and thereby $\operatorname{deg}(\mathcal{I}, \prec) \leq d-1+\prod_{i=1}^{n-1}\left(\operatorname{deg}\left(\mathcal{I}_{i}, \prec\right)+1\right)$.

Set $B_{1}=d$ and for $i \geq 2$ recursively $B_{i}=d-1+$ $\prod_{j=1}^{i-1}\left(B_{j}+1\right)$. If we assume that for each index $1 \leq i<n$ the reduced ideal $\mathcal{I}_{i}$ satisfies $\mathrm{CP}$ w.r.t. $d$, then by the considerations above $\operatorname{deg}\left(\mathcal{I}_{i}, \prec\right) \leq B_{i}$. In particular, $B_{2}=2 d$ and $\operatorname{deg}(\mathcal{I}, \prec) \leq B_{n}$. One easily sees that the $B_{i}$ satisfy the recursion relation $B_{i}=d-1+\left(B_{i-1}+1\right)\left(B_{i-1}-d+1\right)=$ $B_{i-1}^{2}-(d-2) B_{i-1}$ for all $i \geq 2$. Since we may suppose that $d \geq 2$, we have $B_{i} \leq B_{i-1}^{2}$. Thus, for all $i \geq 2$ we have $B_{i} \leq(2 d)^{2^{i-2}}$ and therefore $B_{n}=\operatorname{deg}(\mathcal{I}, \prec) \leq(2 d)^{2^{n-2}}$. We summarize the above discussion in the next theorem.

THEOREM 4.1. ([8]) Suppose that $\mathcal{I}$ is in quasi stable position and that the ideals $\mathcal{I}_{1}, \ldots, \mathcal{I}_{n-1}, \mathcal{I}$ satisfy $C P$ w.r.t. d. Then $\operatorname{deg}(\mathcal{I}, \prec) \leq \operatorname{reg}(\mathcal{I}) \leq(2 d)^{2^{n-2}}$.

Proof. We mentioned already above that for any ideal in quasi stable position $\operatorname{deg}(\mathcal{I}, \prec) \leq \operatorname{reg}(\mathcal{I})$, since the regularity equals the maximal degree of an element of the Pommaret basis of $\mathcal{I}$. As the regularity remains invariant under linear coordinate transformations, we may w.l.o.g. assume that $\mathcal{I}$ is even in strongly stable position where $\operatorname{deg}(\mathcal{I}, \prec)=\operatorname{reg}(\mathcal{I})$ and where Prop. 3.6 entails that also $\mathcal{I}_{1}, \ldots, \mathcal{I}_{n-1}, \mathcal{I}$ satisfy CP w.r.t. $d$. Now the assertion follows from the consideration above.

We derive now a dimension dependent upper bound for $\operatorname{deg}(\mathcal{I}, \prec)$.

TheOrem 4.2. Suppose that $\mathcal{I}$ is in strongly stable position and $D=\operatorname{dim}(\mathcal{I}) \geq 1$. Then

$$
\operatorname{deg}(\mathcal{I}, \prec)=\operatorname{reg}(\mathcal{I}) \leq\left(d^{n-D}+(n-D)(d-1)\right)^{2^{D-1}}
$$

Proof. Since $\mathcal{I}$ is in strongly stable position, the ideal $\mathcal{I}_{n-D} \subset \mathbb{k}\left[x_{1}, \ldots, x_{n-D}\right]$ is zero-dimensional [34, Prop 3.15]. According to Lem. 3.11 $\operatorname{deg}\left(\mathcal{I}_{n-D}\right) \leq(n-D)(d-1)+1$. Hence the maximal degree of a term in $G(\operatorname{LT}(\mathcal{I}))$ which depends only on $x_{1}, \ldots, x_{n-D}$ is at most this bound. We shall now construct an upper bound for the degree of the terms in $G(\operatorname{LT}(\mathcal{I}))$ containing at least one of the remaining variables $x_{n-D+1}, \ldots, x_{n}$. Following the approach of Caviglia and Sbarra, we first look for an upper bound for the number of these terms.

Consider a term $m=x_{1}^{\alpha_{1}} \cdots x_{n}^{\alpha_{n}} \in G(\operatorname{LT}(\mathcal{I}))$ with $\alpha_{i}>0$ for some $i \geq n-D+1$. It is clear that $x_{1}^{\alpha_{1}} \cdots x_{n-D}^{\alpha_{n-D}}$ belongs to the complement of $\operatorname{LT}\left(\mathcal{I}_{n-D}\right)$. Since the ideal $\mathcal{I}_{n-D} \subset \mathbb{k}\left[x_{1}, \ldots, x_{n-D}\right]$ is zero-dimensional, Lem. 3.11 entails that $\operatorname{dim}_{\mathbb{k}}\left(\mathbb{k}\left[x_{1}, \ldots, x_{n-D}\right] / \mathcal{I}_{n-D}\right) \leq d^{n-D}$. Hence the number of terms $x_{1}^{\alpha_{1}} \cdots x_{n-D}^{\alpha_{n-D}}$ is at most $d^{n-D}$. On the other hand, for any index $n-D+1 \leq i \leq n$ we have $\alpha_{i} \leq \operatorname{deg}_{i}(\operatorname{LT}(\mathcal{I})) \leq \operatorname{deg}\left(\mathcal{I}_{i}, \prec\right)$. Furthermore, we know that two distinct term in $G(\operatorname{LT}(\mathcal{I}))$ differ already in their first $n-1$ variables. These arguments imply that the number of terms in $G(\operatorname{LT}(\mathcal{I}))$ containing at least one of the variables $x_{n-D+1}, \ldots, x_{n}$ is at most $d^{n-D} \prod_{i=n-D+1}^{n-1}\left(\operatorname{deg}\left(\mathcal{I}_{i}, \prec\right)+1\right)$.

The strongly stability of $\mathcal{I}$ implies that CP holds for $\operatorname{LT}(\mathcal{I})$ w.r.t. $(n-D)(d-1)+1 \geq d$ by Prop. 3.6. Hence $\operatorname{deg}(\mathcal{I}, \prec)-$ $((n-D)(d-1)+1)+1$ must be less than or equal to the number of terms in $G(\operatorname{LT}(\mathcal{I}))$ containing at least one of the variables $x_{n-D+1}, \ldots, x_{n}$ leading to the estimate

$\operatorname{deg}(\mathcal{I}, \prec) \leq d^{n-D} \prod_{i=n-D+1}^{n-1}\left(\operatorname{deg}\left(\mathcal{I}_{i}, \prec\right)+1\right)+(n-D)(d-1)$

Set $B_{n-D+1}=d^{n-D}+(n-D)(d-1)$ and recursively $B_{j}=$ $d^{n-D} \prod_{i=n-D+1}^{j-1}\left(B_{i}+1\right)+(n-D)(d-1)$ for $n-D+2 \leq j \leq n$. One easily verifies that these numbers satisfy the recursion relation $B_{j}=\left(B_{j-1}-(n-D)(d-1)\right)\left(B_{j-1}+1\right)+(n-D)(d-$ $1)=B_{j-1}^{2}-((n-D)(d-1)-1) B_{j-1}$. We may again assume that $d \geq 2$, and therefore $B_{j} \leq B_{j-1}^{2}$ for $n-D+2 \leq j \leq n$. This implies that $B_{j} \leq\left(d^{n-D}+(n-D)(d-1)\right)^{2^{j-n+D-1}}$ and in particular we have $B_{n} \leq\left(d^{n-D}+(n-D)(d-1)\right)^{2^{D-1}}$.

REMARK 4.3. Let us compare the dimension dependent bounds $A(n, d, D)=2 d^{(n-D) 2^{D-1}}$ derived in Thm. 3.12 and $B(n, d, D)=2\left(1 / 2 d^{n-D}+d\right)^{2^{D-1}}$ due to Mayr and Ritscher [29] with $C(n, d, D)=\left(d^{n-D}+(n-D)(d-1)\right)^{2^{D-1}}$ obtained now. Obviously, all three bounds describe essentially the same qualitative behaviour, although they are derived with fairly different approaches. However, the bound $B(n, d, D)$ of Mayr and Ritscher has almost always the best constants. But there are some cases where one of the other bounds is better. For example, in the case of a hypersurface, i.e. for $D=n-1, A(n, d, D)$ is smaller than $B(n, d, D)$. For some curves of low degree, i.e. for $D=1$ and small values of $d, C(n, d, D)$ is smaller than $B(n, d, D)$. Some concrete inequalities are:

$$
\begin{aligned}
& \text { - } A(5,3,4)<C(5,3,4), \\
& \text { - } A(3,5,2)>C(3,5,2), \\
& \text { - } A(5,2,4)<B(5,2,4), \\
& \text { - } A(5,4,2)>B(5,4,2), \\
& \text { - } B(4,5,1)>C(4,5,1), \\
& \text { - } B(5,2,3)<C(5,2,3) .
\end{aligned}
$$

Hence no bound is always the best one. 
Again an application of Prop. 3.1 yields immediately an improved bound depending on both the depth and the dimension of $\mathcal{I}$.

COROLlary 4.4. Under the assumptions of Thm. 4.2, one has $\operatorname{deg}(\mathcal{I}, \prec)=\operatorname{reg}(\mathcal{I}) \leq\left(d^{n-D}+(n-D)(d-1)\right)^{2^{D-\lambda}-1}$.

It should be noted that in positive characteristic it is not always possible to achieve strongly stable position by linear coordinate transformations (see 21 for a more detailed discussion). Nevertheless, following [8, we state the following conjecture.

Conjecture 4.5. The upper bound for the CastelnuovoMumford regularity of $\mathcal{I}$ in Cor. 4.4 holds independently of the characteristic of $\mathbb{k}$.

\section{LAZARD'S UPPER BOUND}

Finally, in this section we study Lazard's upper bound 23 for the degree of Gröbner bases for both homogeneous and non-homogeneous ideals. We provide a simple proof for his results and generalize Giusti's bound to non-homogeneous ideals. Note that for Lazard 23] dimension was always the one as projective variety, whereas we use throughout this paper the one as affine variety which is one higher. In the sequel, we always set $d_{i}=1$ for any $i>k$.

Theorem 5.1. ([23, Thm. 2]) Assume that $\operatorname{dim}(\mathcal{I}) \leq 1$. Then we have $\operatorname{deg}(\operatorname{gin}(\mathcal{I}), \prec) \leq d_{1}+\cdots+d_{r}-r+1$ where $r=n-\lambda$.

We showed in 20 that many properties of $\operatorname{gin}(\mathcal{I})$ also hold for $\operatorname{lt}(\mathcal{I})$ provided $\mathcal{I}$ is in quasi stable position. Along these lines, we shall now prove that in Lazard's upper bound we can replace $\operatorname{gin}(\mathcal{I})$ by $\mathcal{I}$, if $\mathcal{I}$ is in quasi stable position. For this, we need the next proposition also due to Lazard, which is the key point in the proof of the above theorem.

Proposition $5.2 \quad$ ([22, ThM. 3.3]). Assume again that $\operatorname{dim}(\mathcal{I}) \leq 1$. Then $\operatorname{dim}_{\mathbb{k}}(\mathcal{P} / \mathcal{I})_{\ell}=\operatorname{dim}_{\mathbb{k}}(\mathcal{P} / \mathcal{I})_{\ell+1}$ for each $\ell \geq d_{1}+\cdots+d_{n}-n+1$.

Thus, under the assumptions of this proposition, we can say that $\operatorname{hilb}(\mathcal{I}) \leq d_{1}+\cdots+d_{n}-n+1$.

TheOREM 5.3. Suppose that $\mathcal{I}$ is in quasi stable position and $\operatorname{dim}(\mathcal{I}) \leq 1$. Then, $\operatorname{deg}(\mathcal{I}, \prec) \leq d_{1}+\cdots+d_{r}-r+1$ where $r=n-\lambda$.

Proof. It suffices to show that $\operatorname{deg}(\mathcal{I}, \prec) \leq d_{1}+\cdots+$ $d_{n}-n+1$, since then the desired inequality follows immediately from Prop. 3.1 As $\mathcal{I}$ is in quasi stable position, we have the inequality $\operatorname{deg}(\mathcal{I}, \prec) \leq \max \left\{\operatorname{hilb}(\mathcal{I}), \operatorname{hilb}\left(\mathcal{I}^{\prime}\right)\right\}$ where $\mathcal{I}^{\prime}=\left(\mathcal{I}+\left\langle x_{n}\right\rangle\right) \cap \mathbb{k}\left[x_{1}, \ldots, x_{n-1}\right]$ is an ideal in the ring $\mathbb{k}\left[x_{1}, \ldots, x_{n-1}\right]$ [19, Thm. 4.17], 36, Thm. 4.7]. Obviously, $\mathcal{I}^{\prime}$ is generated by $\left.f_{1}\right|_{x_{n}=0}, \ldots,\left.f_{k}\right|_{x_{n}=0}$ and $\operatorname{dim}(\mathcal{I}) \leq 1$ (by using the fact that $\mathcal{I}$ is in quasi stable position) entails $\operatorname{dim}\left(\mathcal{I}^{\prime}\right) \leq 1$. These arguments show that, by Prop. 5.2 $\operatorname{hilb}(\mathcal{I}) \leq d_{1}+\cdots+d_{n}-n+1$ and $\operatorname{hilb}\left(\mathcal{I}^{\prime}\right) \leq d_{1}+\cdots+$ $d_{n-1}-(n-1)+1$ which proves the assertion.

ExAmple 5.4. Lazard [23, Conj. 3] conjectured that the conclusion of Theorem 5.1 remained true, if one replaces $\operatorname{gin} \mathcal{I}$ by $\mathcal{I}$. Mora claimed that the following ideal (see the Appendix of [23]) provided a counter-example. Consider the homogeneous ideal $\mathcal{I}=\left\langle x_{1} x_{2}^{t-1}-x_{3}^{t}, x_{1}^{t+1}-x_{2} x_{3}^{t-1} x_{4}, x_{1}^{t} x_{3}-\right.$ $\left.x_{2}^{t} x_{4}\right\rangle$ in the polynomial ring $\mathcal{P}=\mathbb{k}\left[x_{1}, \ldots, x_{4}\right]$. Thus we have $d_{1}=t, d_{2}=d_{3}=t+1$. One can show that the polynomial $x_{3}^{t^{2}+1}-x_{2}^{t^{2}} x_{4}$ appears in the Gröbner basis of $\mathcal{I}$ and hence $\operatorname{deg}(\mathcal{I}, \prec) \geq t^{2}+1$. For simplicity we restrict to the case $t=4$ where we obtain

$$
\operatorname{LT}(\mathcal{I})=\left\langle x_{1} x_{2}^{3}, x_{1}^{4} x_{3}, x_{1}^{5}, x_{1}^{3} x_{3}^{5}, x_{1}^{2} x_{3}^{9}, x_{1} x_{3}^{13}, x_{3}^{17}\right\rangle .
$$

Thus we find here $\operatorname{deg}(\mathcal{I}, \prec)=17>d_{1}+d_{2}+d_{3}-3=11$. But as $\operatorname{dim}(\mathcal{I})=2$, $\mathcal{I}$ does not yield a counter-example to Lazard's conjecture. However, if we consider $\mathcal{I}^{\prime}=\left.\mathcal{I}\right|_{x_{4}=0} \subset$ $\mathbb{k}\left[x_{1}, x_{2}, x_{3}\right]$, then we find that $\mathcal{I}^{\prime}$ has dimension 1 and that $\mathrm{LT}\left(\mathcal{I}^{\prime}\right)$ is generated by the same terms as $\mathrm{LT}(\mathcal{I}) . \mathcal{I}^{\prime}$ is not in quasi stable position, as no pure power of $x_{2}$ belongs to $\operatorname{LT}\left(\mathcal{I}^{\prime}\right)$. Hence $\mathcal{I}^{\prime}$ represents a counter-example to Lazard's conjecture. This example shows furthermore that in Thm. 5.3 it is not possible to drop the assumption of quasi stable position.

REMARK 5.5. We gave above a direct proof for Thm. 5.3 However, we can provide a more concise proof using Thm. 5.1 and Pommaret bases. Indeed, from Thm.5.1 it follows that $\operatorname{reg}(\mathcal{I}) \leq d_{1}+\cdots+d_{r}-r+1$ where $r=n-\lambda$, as $\operatorname{reg}(\mathcal{I})=$ $\operatorname{deg}(\operatorname{gin}(\mathcal{I}), \prec)$. Since the ideal $\mathcal{I}$ is in quasi stable position, it possesses a finite Pommaret basis $\mathcal{H}$ where $\operatorname{reg}(\mathcal{I})$ is the maximal degree of the elements of $\mathcal{H}$ and therefore $\operatorname{deg}(\mathcal{I}, \prec) \leq d_{1}+\cdots+d_{r}-r+1$. These considerations also yield immediately the following corollary.

Corollary 5.6. If $\operatorname{dim}(\mathcal{I}) \leq 1$, then $\operatorname{reg}(\mathcal{I}) \leq d_{1}+\cdots+$ $d_{r}-r+1$ where $r=n-\lambda$.

Finally, we present an affine version of Thm.5.3. We drop now the assumption that the polynomials $f_{1}, \ldots, f_{k}$ generating $\mathcal{I}$ are homogeneous. Let $x_{n+1}$ be an extra variable and $\tilde{f}$ the homogenization of $f$ using $x_{n+1}$. We further denote by $\tilde{\mathcal{I}}$ the ideal generated by $\tilde{f}_{1}, \ldots, \tilde{f}_{k}$ (note that in general this is not equal to the homogenization of $\mathcal{I}$ ). The next proposition may be considered as a generalization of Lazard's upper bound [23, Thm. 2] to ideals in quasi stable position.

Proposition 5.7. Assume that $\tilde{\mathcal{I}}$ is in quasi stable position, that $\operatorname{dim}(\tilde{\mathcal{I}}) \leq 1$ and that $\operatorname{depth}(\tilde{\mathcal{I}})=\lambda$. Then, $\operatorname{deg}(\mathcal{I}, \prec) \leq d_{1}+\cdots+d_{r}-r+1$ where $r=n+1-\lambda$.

Proof. By Thm. 5.3, hilb $(\tilde{\mathcal{I}}) \leq \operatorname{reg}(\tilde{\mathcal{I}}) \leq d_{1}+\cdots+d_{r}-$ $r+1$ where $r=n+1-\lambda$. Hence $\operatorname{dim}_{\mathbb{k}}\left(\mathbb{k}\left[x_{1}, \ldots, x_{n+1}\right] / \tilde{\mathcal{I}}\right)_{\ell}=$ $\operatorname{dim}_{\mathbb{k}}\left(\mathbb{k}\left[x_{1}, \ldots, x_{n+1}\right] / \tilde{\mathcal{I}}\right)_{\ell+1}$ for all degrees $\ell \geq d_{1}+\cdots+d_{r}-$ $r+1$. Therefore, we have $\operatorname{dim}_{\mathbb{k}}(\mathcal{P} / \mathcal{I})_{\leq \ell}=\operatorname{dim}_{\mathbb{k}}(\mathcal{P} / \mathcal{I})_{\leq \ell+1}$ for each $\ell \geq d_{1}+\cdots+d_{r}-r+1$ and this observation implies that the reduced Gröbner basis of $\mathcal{I}$ contains no element of degree greater than $d_{1}+\cdots+d_{r}-r+1$.

We conclude this paper by mentioning that it is easy to see that for a homogeneous ideal with $\operatorname{dim}(\mathcal{I}) \leq 1$, being in quasi stable position is equivalent to being in Nother position. This implies that in Thm. 5.3 and Prop. 5.7 one can replace "quasi stable position" by "Nœther position".

\section{Acknowledgments.}

The research of the first author was in part supported by a grant from IPM (No. 94550420). The work of the sec- 
ond author was partially performed as part of the H2020FETOPEN-2016-2017-CSA project $S C^{2}$ (712689). The authors would like to thank the anonymous reviewers for their valuable comments.

\section{REFERENCES}

[1] T. Becker and V. Weispfenning. Gröbner Bases, a Computational Approach to Commutative Algebra. Springer, 1993.

[2] D. Bayer. The Division Algorithm and the Hilbert Scheme. Ph.D. thesis, Harvard University, 1982.

[3] D. Bayer and M. Stillman. A Criterion for Detecting m-Regularity. Invent. Math. 87(1), pages 1-11, 1987.

[4] I. Bermejo and P. Gimenez. Computing the Castelnuovo-Mumford Regularity of Some Subschemes of $\mathbb{P}_{K}^{n}$ Using Quotients of Monomial Ideals. J. Pure Appl. Algebra, 164(1-2), pages 23-33, 2001.

[5] I. Bermejo and Ph. Gimenez. Saturation and Catelnuovo-Mumford regularity. J. Algebra, 303, pages 592-617, 2006.

[6] B. Buchberger. Ein Algorithmus zum Auffinden der Basiselemente des Restklassenringes nach einem nulldimensionalen Polynomideal. PhD thesis, Universität Innsbruck, 1965.

[7] B. Buchberger. A Criterion for Detecting Unnecessary Reductions in the Construction of Gröbner Bases. EUROSAM'r9, Lecture Notes in Compute. Sci., Springer, 72, pages 3-21, 1979.

[8] G. Caviglia and E. Sbarra. Characteristic-free Bounds for the Castelnuovo-Mumford Regularity. Compos. Math., 141(6), pages 1365-1373, 2005.

[9] D. Cox, J. Little and D. O'Shea. Ideals, Varieties, and Algorithms. Springer-Verlag, third edition, 2007.

[10] T. Dubé. The Structure of Polynomial Ideals and Gröbner Bases. SIAM Journal on Computing, 19, pages 750-773, 1990.

[11] D. Eisenbud. Commutative Algebra with a View toward Algebraic Geometry. Springer-Verlag, 1995.

[12] D. Eisenbud and S. Goto. Linear Free Resolutions and Minimal Multiplicity. J. Algebra, 88(1), pages 89-133, 1984.

[13] R. Fröberg. An Introduction to Gröbner Bases. John Wiley \& Sons Ltd., Chichester, 1997.

[14] A. Galligo. A propos du théorème de preparation de Weierstrass. Lect. Notes Math., 409, pages 543-579, 1974.

[15] A. Galligo. Théorème de division et stabilité en géométrie analytique locale.. Ann. Inst. Fourier, 29(2), pages 107-184, 1979.

[16] M. Giusti. Some Effectivity Problems in Polynomial Ideal Theory. EUROSAM'84, Lecture Notes in Comput. Sci., 174, pages 159-171, Springer, 1984.

[17] M. Green. Generic Initial Ideals. In: Elias, J., Giral, J., Miró-Roig, R., Zarzuela, S. (eds.) Six Lectures on Commutative Algebra, pages 119-186. Progress in Mathematics 166, Birkhäuser, Basel, 1998.

[18] G.M. Greuel and G. Pfister. A Singular Introduction to Commutative Algebra. Springer-Verlag, 2008.

[19] A. Hashemi. Strong Noether Position and Stabilized Regularities. Commun. Algebra, 38(2), pages 515-533, 2010 .
[20] A. Hashemi, M. Schweinfurter and W.M. Seiler. Quasi-stability versus Genericity. Proc. CASC'12, Lecture Notes in Comput. Sci., 7442, pages 172-184, 2012.

[21] A. Hashemi, M. Schweinfurter and W.M. Seiler. Deterministic Genericity for Polynomial Ideals. $J$. Symb. Comput., accepted for publication (2017), 31 pages (doi.org/10.1016/j.jsc.2017.03.008).

[22] D. Lazard. Résolution des systèmes d'équations algébriques. Theoret. Comput. Sci. 15(1), pages 77-110, 1981.

[23] D. Lazard. Gröbner Bases, Gaussian Elimination and Resolution of Systems of Algebraic Equations. Proc. EUROCAL'83, Lecture Notes in Comput. Sci., 162, pages 146-156, Springer, 1983.

[24] D. Lazard. A Note on Upper Bounds for Ideal-Theoretic Problems. J. Symb. Comput., 13(3), pages 231-234, 1992.

[25] M. Lejeune-Jalabert. Effectivité de calculs polynomiaux. Cours de D.E.A, Institute Fourier, Grenoble. 1984.

[26] D. Mall. On the Relation between Gröbner and Pommaret Bases. Appl. Alg. Eng. Comm. Comp., 9, pages $117-123,1998$.

[27] H. Matsumura. Commutative ring theory. Cambridge University Press, second edition, 1989.

[28] E. W. Mayr and A. R. Meyer. The Complexity of the Word Problems for Commutative Semigroups and Polynomial Ideals. Adv. Math., 46(3), pages 305-329, 1982.

[29] E.W. Mayr and S. Ritscher. Dimension-Dependent Bounds for Gröbner Bases of Polynomial Ideals. J. Symb. Comput., 49, pages 78-94, 2013.

[30] H.M. Möller and F. Mora. Upper and Lower Bounds for the Degree of Groebner Bases. In Proc. EUROSAM'84, Lecture Notes in Comput. Sci., 174, pages 172-183. Springer, 1984.

[31] T. Mora. Solving Polynomial Equation Systems II: Macaulay's Paradigm and Gröbner Technology. Cambridge University Press, 2005.

[32] D. Mumford. Lectures on Curves on an Algebraic Surface. Princeton University Press, 1966.

[33] W.M. Seiler. A Combinatorial Approach to Involution and $\delta$-Regularity I: Involutive Bases in Polynomial Algebras of Solvable Type. Appl. Alg. Eng. Comm. Comp. 20, pages 207-259, 2009.

[34] W.M. Seiler. A Combinatorial Approach to Involution and $\delta$-Regularity II: Structure Analysis of Polynomial Modules with Pommaret Bases. Appl. Alg. Eng. Comm. Comp. 20, pages 261-338, 2009.

[35] W.M. Seiler. Involution - The Formal Theory of Differential Equations and its Applications in Computer Algebra. Springer-Verlag, 2010.

[36] W.M. Seiler. Effective Genericity, $\delta$-Regularity and Strong Noether Position. Commun. Algebra, 40(10), pages 3933-3949, 2012.

[37] C. Yap. A New Lower Bound Construction for the Word Problem for Commutative Thue Systems. J. Symb. Comput., 12(1), pages 1-28, 1991. 